\title{
On 2-Absorbing Primary Fuzzy Ideals of Commutative Rings
}

\author{
Deniz Sönmez, ${ }^{1}$ Gürsel Yeşilot, ${ }^{1}$ Serkan Onar, ${ }^{1}$ Bayram Ali Ersoy, ${ }^{1}$ and Bijan Davvaz ${ }^{2}$ \\ ${ }^{1}$ Department of Mathematics, Yildiz Technical University, Davutpaşa, Istanbul, Turkey \\ ${ }^{2}$ Department of Mathematics, Yazd University, Yazd, Iran \\ Correspondence should be addressed to Deniz Sönmez; dnzguel@hotmail.com
}

Received 6 April 2017; Accepted 20 July 2017; Published 23 August 2017

Academic Editor: Leonid Shaikhet

Copyright (c) 2017 Deniz Sönmez et al. This is an open access article distributed under the Creative Commons Attribution License, which permits unrestricted use, distribution, and reproduction in any medium, provided the original work is properly cited.

In this work, we give a characterization of generalizations of prime and primary fuzzy ideals by introducing 2-absorbing fuzzy ideals and 2-absorbing primary fuzzy ideals and establish relations between 2-absorbing (primary) fuzzy ideals and 2-absorbing (primary) ideals. Furthermore, we give some fundamental results concerning these notions.

\section{Introduction}

The fundamental concept of fuzzy set was introduced by Zadeh [1] in 1965. In 1982, Liu introduced the notion of fuzzy ideal of a ring [2]. Mukherjee and Sen have continued the study of fuzzy ideals by introducing the notion of prime fuzzy ideals [3]. To the present day, fuzzy algebraic structures have been developed and many interesting results were obtained.

Prime ideals and primary ideals play a significant role in commutative ring theory. Because of this importance, the concept of 2-absorbing ideals, which is a generalization of prime ideals [4], and the concept of 2-absorbing primary ideals, which is a generalization of primary ideals [5], were introduced. While the prime fuzzy ideals and primary fuzzy ideals have been investigated $[3,6]$, the concepts of 2absorbing fuzzy ideals and 2-absorbing primary fuzzy ideals have not been studied yet. In this paper, we introduce the 2absorbing fuzzy ideals and 2-absorbing primary fuzzy ideals and some generalizations of 2-absorbing primary fuzzy ideals and describe some properties of 2-absorbing primary fuzzy ideals.

Let $R$ be a commutative ring with identity. Recall that a proper ideal $I$ of $R$ is called a 2-absorbing ideal if whenever $a, b, c \in R$ and $a b c \in I$, then either $a b \in I$ or $a c \in I$ or $b c \in I$ [4] and a proper ideal $I$ of $R$ is called a 2-absorbing primary ideal if whenever $a, b, c \in R$ and $a b c \in I$, then either $a b \in I$ or $a c \in \sqrt{I}$ or $b c \in \sqrt{I}$ [5]. Based on these definitions, a nonconstant fuzzy ideal $\mu$ of $R$ is called a 2-absorbing fuzzy ideal of $R$ if for any fuzzy points $x_{r}, y_{s}, z_{t}$ of $R, x_{r} y_{s} z_{t} \in \mu$ implies that either $x_{r} y_{s} \in \mu$ or $x_{r} z_{t} \in \mu$ or $y_{s} z_{t} \in \mu$ and a nonconstant fuzzy ideal $\mu$ of $R$ is said to be a 2-absorbing primary fuzzy ideal of $R$ if for any fuzzy points $x_{r}, y_{s}, z_{t}$ of $R, x_{r} y_{s} z_{t} \in \mu$ implies that either $x_{r} y_{s} \in \mu$ or $x_{r} z_{t} \in \sqrt{\mu}$ or $y_{s} z_{t} \in \sqrt{\mu}$. It is shown that if $\mu$ is a 2 -absorbing primary fuzzy ideal of $R$, then $\sqrt{\mu}$ is 2-absorbing fuzzy ideal. We introduce the notions of weakly completely 2-absorbing fuzzy ideal and weakly completely 2-absorbing primary fuzzy ideal, which is a weakened status of the 2-absorbing fuzzy ideals and 2absorbing primary fuzzy ideal, respectively. Then relationship between the 2-absorbing primary fuzzy ideals and weakly completely 2-absorbing primary fuzzy ideals is analyzed. Based on the definition of the level set, the transition of 2-absorbing primary ideals of $R$ and 2-absorbing primary fuzzy ideals of $R$ is examined. By a 2 -absorbing primary ideal of $R$, a 2-absorbing primary fuzzy ideal is established (Proposition 20). For a ring homomorphism $f: R \rightarrow S$, it is shown that $f^{-1}(\xi)$ is a 2 -absorbing primary fuzzy ideal of $R$, where $\xi$ is 2-absorbing primary fuzzy ideal of $S$. If $\mu$ is 2 -absorbing primary fuzzy ideal of $R$, which is constant on Ker $f$, then it is proved that $f(\mu)$ is a 2-absorbing primary fuzzy ideal of $S$. It is shown under what condition the intersection of the collection of 2-absorbing primary fuzzy ideals is 2-absorbing primary fuzzy ideal. It is shown that the intersection of two 2-absorbing primary fuzzy ideals need not be a 2-absorbing primary fuzzy ideal if this condition is not satisfied (Example 27). Also, it is proved that union of a 
directed collection of 2-absorbing primary fuzzy ideals of $R$ is 2-absorbing primary fuzzy ideal.

\section{Preliminaries}

We assume throughout that all rings are commutative with $1 \neq 0$. Unless stated otherwise $L=[0,1]$ stands for a complete lattice. $Z$ denotes the ring of integers, $L(R)$ denotes the set of fuzzy sets of $R$, and $L I(R)$ denotes the set of fuzzy ideals of $R$. For $\mu, \xi \in L(R)$, we say $\mu \subseteq \xi$ if and only if $\mu(x) \leq \xi(x)$ for all $x \in R$. When $r \in L, x, y \in R$ we define $x_{r} \in L(R)$ as follows:

$$
x_{r}(y)= \begin{cases}r & x=y \\ 0 & \text { otherweise }\end{cases}
$$

and $x_{r}$ is referred to as fuzzy point of $R$.

Also, for $\mu \in L(R)$ and $t \in L$, define $\mu_{t}$ as follows:

$$
\mu_{t}=\{x \in R: \mu(x) \geq t\} .
$$

Definition 1 (see [2]). A fuzzy subset $\mu$ of a ring $R$ is called a fuzzy ideal of $R$ if for all $x, y \in R$ the following conditions are satisfied:

(i) $\mu(x-y) \geq \mu(x) \wedge \mu(y), \forall x, y \in R$.

(ii) $\mu(x y) \geq \mu(x) \vee \mu(y), \forall x, y \in R$.

Let $\mu$ be any fuzzy ideal of $R ; x, y \in R$, and let 0 be the additive identity of $R$. Then it is easy to verify the following:

(i) $\mu(0) \geq \mu(x), \mu(x)=\mu(-x)$ and $\mu_{t} \subset \mu_{s}$, where $s, t \in$ $\operatorname{Im}(\mu)$ and $t>s$.

(ii) If $\mu(0)=\mu(x-y)$, then $\mu(x)=\mu(y), \mu(x)=s$ iff $x \in \mu_{s}$, and $x \notin \mu_{t}, \forall t>s$.

Definition 2 (see [7]). Let $\mu$ be any fuzzy ideal of $R$. The ideals $\mu_{t},(\mu(0) \geq t)$ are called level ideals of $\mu$.

Definition 3 (see [3]). A fuzzy ideal $\mu$ of $R$ is called prime fuzzy ideal if for any two fuzzy points $x_{r}, y_{s}$ of $R, x_{r} y_{s} \in \mu$ implies either $x_{r} \in \mu$ or $y_{s} \in \mu$.

Definition 4 (see [6]). Let $\mu$ be a fuzzy ideal of $R$. Then $\sqrt{\mu}$, called the radical of $\mu$, is defined by $\sqrt{\mu}(x)=\bigvee_{n \geq 1} \mu\left(x^{n}\right)$.

Definition 5 (see [6]). A fuzzy ideal $\mu$ of $R$ is called primary fuzzy ideal if for $x, y \in R, \mu(x y)>\mu(x)$ implies $\mu(x y) \leq$ $\mu\left(y^{n}\right)$ for some positive integer $n$.

Theorem 6 (see [6]). Let $\mu$ be fuzzy ideal of a ring $R$. Then $\sqrt{\mu}$ is a fuzzy ideal of $R$.

Definition 7 (see [3]). Let $R$ be a ring. Then a nonconstant fuzzy ideal $\mu$ is said to be weakly completely prime fuzzy ideal iff for $x, y \in R, \mu(x y)=\max \{\mu(x), \mu(y)\}$.

Theorem 8 (see [8]). If $\mu$ and $\xi$ are two fuzzy ideals of $R$, then $\sqrt{\mu \cap \xi}=\sqrt{\mu} \cap \sqrt{\xi}$.
Theorem 9 (see [8]). Let $f: R \rightarrow S$ be a ring homomorphism and let $\mu$ be a fuzzy ideal of $R$ such that $\mu$ is constant on Ker $f$ and let $\xi$ be a fuzzy ideal of S. Then,

(i) $\sqrt{f(\mu)}=f(\sqrt{\mu})$,

(ii) $\sqrt{f^{-1}(\xi)}=f^{-1}(\sqrt{\xi})$.

Definition 10 (see [4]). A nonzero proper ideal $I$ of a commutative ring $R$ with $1 \neq 0$ is called a 2 -absorbing ideal if whenever $a, b, c \in R$ with $a b c \in I$, then either $a b \in I$ or $a c \in I$ or $b c \in I$.

Definition 11 (see [5]). A proper ideal $I$ of $R$ is called a 2absorbing primary ideal of $R$ if whenever $a, b, c \in R$ with $a b c \in I$, then either $a b \in I$ or $a c \in \sqrt{I}$ or $b c \in \sqrt{I}$.

Theorem 12 (see [5]). If I is a 2-absorbing primary ideal of $R$, then $\sqrt{I}$ is a 2-absorbing ideal of $R$.

Definition 13 (see [9]). An element $1>\alpha \in L$ is called a 2absorbing element if for any $x, y, z \in L, x \wedge y \wedge z<\alpha$ implies either $x \wedge y<\alpha$ or $x \wedge z<\alpha$ or $y \wedge z<\alpha$.

\section{2-Absorbing Primary Fuzzy Ideals}

Definition 14. Let $\mu$ be a nonconstant fuzzy ideal of $R$. Then $\mu$ is called a 2-absorbing fuzzy ideal of $R$ if for any fuzzy points $x_{r}, y_{s}, z_{t}$ of $R, x_{r} y_{s} z_{t} \in \mu$ implies that either $x_{r} y_{s} \in \mu$ or $x_{r} z_{t} \in$ $\mu$ or $y_{s} z_{t} \in \mu$.

Theorem 15. Every prime fuzzy ideal of $R$ is a 2-absorbing fuzzy ideal.

Proof. The proof is straightforward.

Lemma 16. Let $\mu$ be a fuzzy ideal and $t \in[0, \mu(0)]$. If $\mu$ is a 2-absorbing fuzzy ideal, then $\mu_{t}$ is a 2-absorbing ideal of $R$.

Proof. Let $\mu$ be a 2-absorbing fuzzy ideal and $t \in[0, \mu(0)]$. If $x y z \in \mu_{t}$ for any $x, y, z \in R$, then $\mu(x y z) \geq t$. Thus, $(x y z)_{t}(x y z)=t \leq \mu(x y z)$ and $(x y z)_{t}=x_{t} y_{t} z_{t} \in \mu$. Since $\mu$ is a 2-absorbing fuzzy ideal, we have $x_{t} y_{t}=(x y)_{t} \in \mu$ or $x_{t} z_{t}=(x z)_{t} \in \mu$ or $y_{t} z_{t}=(y z)_{t} \in \mu$. Hence, $x y \in \mu_{t}$ or $x z \in \mu_{t}$ or $y z \in \mu_{t}$. Therefore, $\mu_{t}$ is a 2 -absorbing ideal of $R$.

The following example shows that the converse of the lemma need not be true.

Example 17. Let $R=Z$, the ring of integers. Define the fuzzy ideal $\mu$ of $Z$ by

$$
\mu(x)= \begin{cases}1, & x=0, \\ \frac{1}{4}, & x \in 4 Z-0, \\ 0, & x \in Z-4 Z .\end{cases}
$$

Then $\mu_{t}$ is (0), $4 Z, Z$ in case $t \geq 1, t \geq 1 / 4, t \geq 0$, respectively. Thus, it is seen that $\mu_{t}$ is 2 -absorbing ideal for 
all $t \in \operatorname{Im} \mu$. Since $2_{1 / 2} 2_{1 / 2} 1_{1 / 4}(4)=1 / 4 \leq \mu(4)=1 / 4$ so $2_{1 / 2} 2_{1 / 2} 1_{1 / 4} \in \mu$ but $2_{1 / 2} 2_{1 / 2}(4)=1 / 2>\mu(4)=1 / 4$ and $2_{1 / 2} 1_{1 / 4}(2)=1 / 4>\mu(2)=0$. Hence, $\mu$ is not 2 -absorbing fuzzy ideal.

Definition 18. Let $\mu$ be a nonconstant fuzzy ideal of $R$. Then $\mu$ is said to be a 2-absorbing primary fuzzy ideal of $R$ if $x_{r} y_{s} z_{t} \in$ $\mu$ implies that either $x_{r} y_{s} \in \mu$ or $x_{r} z_{t} \in \sqrt{\mu}$ or $y_{s} z_{t} \in \sqrt{\mu}$ for any fuzzy points $x_{r}, y_{s}, z_{t}$.

Theorem 19. Every primary fuzzy ideal of $R$ is a 2-absorbing primary fuzzy ideal of $R$.

Proof. It is clear from the definition of primary fuzzy ideal.

Proposition 20. Let $I$ be a 2-absorbing primary ideal of $R$ and $1 \neq \alpha \in L$ a 2-absorbing element. If $\mu$ is the fuzzy ideal of $R$ defined by

$$
\mu(x)= \begin{cases}1, & x \in I, \\ \alpha, & x \notin I,\end{cases}
$$

for all $x \in R$, then $\mu$ is a 2-absorbing primary fuzzy ideal of $R$.

Proof. Assume that $x_{r} y_{s} z_{t} \in \mu$ but $x_{r} y_{s} \notin \mu$ and $x_{r} z_{t} \notin \sqrt{\mu}$ and $y_{s} z_{t} \notin \sqrt{\mu}$ for any $x, y, z \in R$. Then $\mu(x y)<r \wedge s$ and $\mu\left((y z)^{n}\right) \leq \sqrt{\mu}(y z)<s \wedge t$ and $\mu\left((x z)^{n}\right) \leq \sqrt{\mu}(x z)<r \wedge t$ for all $n \geq 1$. In this case, $\mu(x y)=\alpha$ and $x y \notin I, \mu\left((y z)^{n}\right)=\alpha$ and $(y z)^{n} \notin I$ so $y z \notin \sqrt{I}, \mu\left((x z)^{n}\right)=\alpha$, and $(x z)^{n} \notin I$ so $x z \notin \sqrt{I}$. Since $I$ is a 2 -absorbing primary ideal of $R$, then we get $x y z \notin I$ and so $\mu(x y z)=\alpha$. By our assumption we get $(x y z)_{r \wedge s \wedge t}=x_{r} y_{s} z_{t} \in \mu$ and $r \wedge s \wedge t \leq \mu(x y z)=\alpha$. Thus, $r \wedge s \leq$ $\alpha$ or $s \wedge t \leq \alpha$ or $r \wedge t \leq \alpha$, since $\alpha$ is 2 -absorbing element, which is a contradiction. Hence, $\mu$ is a 2 -absorbing primary fuzzy ideal of $R$.

Theorem 21. Every 2-absorbing fuzzy ideal of $R$ is a 2absorbing primary fuzzy ideal.

Proof. The proof is straightforward by the definition of the 2-absorbing fuzzy ideal.

The following example shows that the converse of Theorem 21 is not true.

Example 22. Let $\mu \in L I(R)$ be defined as

$$
\mu(x)= \begin{cases}1, & x \in 8 Z, \\ 0, & x \notin 8 Z .\end{cases}
$$

By Theorem 19 and Proposition $20 \mu$ is a 2-absorbing primary fuzzy ideal. For $2 \in Z, 2_{1} 2_{1} 2_{1} \in \mu$ but $22_{1} 2_{1}(4)=1>\mu(4)=0$ so $22_{1} \notin \mu$. Thus, $\mu$ is not a 2 -absorbing fuzzy ideal.

Lemma 23. Let $\mu$ be a fuzzy ideal and $t \in[0, \mu(0)]$. If $\mu$ is a 2absorbing primary fuzzy ideal, then $\mu_{t}$ is a 2-absorbing primary ideal of $R$.
Proof. If $x y z \in \mu_{t}$ for any $x, y, z \in R$, then $\mu(x y z) \geq t$. Thus, $(x y z)_{t}(x y z)=t \leq \mu(x y z)$ and $(x y z)_{t}=x_{t} y_{t} z_{t} \in \mu$. Since $\mu$ is a 2-absorbing primary fuzzy ideal, we have $x_{t} y_{t}=(x y)_{t} \in \mu$ or $x_{t} z_{t}=(x z)_{t} \in \sqrt{\mu}$ or $y_{t} z_{t}=(y z)_{t} \in \sqrt{\mu}$. Hence, $x y \in \mu_{t}$ or $x z \in \sqrt{\mu}_{t}=\sqrt{\mu_{t}}$ or $y z \in \sqrt{\mu}_{t}=\sqrt{\mu_{t}}$. Therefore, $\mu_{t}$ is a 2absorbing primary ideal.

Note that if $\mu_{t}$ is a 2-absorbing primary ideal of $R$, then $\mu$ need not be 2 -absorbing primary fuzzy ideal of $R$. In Example 17, $\mu$ is not 2-absorbing fuzzy ideal and also it is not 2 -absorbing primary fuzzy ideal by Theorem 21 , although $\mu_{t}$ is a 2-absorbing primary ideal of $R$.

Proposition 24. If $\mu$ is a 2-absorbing primary fuzzy ideal of $R$, then $\sqrt{\mu}$ is a 2-absorbing fuzzy ideal of $R$.

Proof. Let $x_{r}, y_{s}, z_{t}$ be any fuzzy points of $R$ such that $x_{r} y_{s} z_{t} \in$ $\sqrt{\mu}$ and $x_{r} y_{s} \notin \sqrt{\mu}$. Since $x_{r} y_{s} z_{t} \in \sqrt{\mu}$, we get $r \wedge s \wedge t=$ $x_{r} y_{s} z_{t}(x y z) \leq \sqrt{\mu}(x y z)$. By the definition of $\sqrt{\mu}, \sqrt{\mu}(x y z)=$ $\bigvee_{n \geq 1} \mu\left(x^{n} y^{n} z^{n}\right) \geq r \wedge s \wedge t$. Then there is a $k \in Z^{+}$such that $r \wedge s \wedge t \leq \mu\left(x^{k} y^{k} z^{k}\right)=\mu\left((x y z)^{k}\right)$. It implies that $\left(x_{r} y_{s} z_{t}\right)^{k} \in \mu$. If $x_{r} y_{s} \notin \sqrt{\mu}$, then, for all $k \in Z^{+},\left(x_{r} y_{s}\right)^{k}=x_{r}^{k} y_{s}^{k} \notin \mu$. Since $\mu$ is a 2-absorbing primary fuzzy ideal, we conclude $x_{r} z_{t} \in \sqrt{\mu}$ or $y_{s} z_{t} \in \sqrt{\mu}$. Thus, $\sqrt{\mu}$ is a 2-absorbing fuzzy ideal.

Definition 25. Let $\mu$ be a 2-absorbing primary fuzzy ideal of $R$. Then $\gamma=\sqrt{\mu}$ is a 2-absorbing fuzzy ideal by Proposition 24 . We say that $\mu$ is a $\gamma$-2-absorbing primary fuzzy ideal of $R$.

Theorem 26. Let $\mu_{1}, \mu_{2}, \ldots, \mu_{n}$ be $\gamma$-2-absorbing primary fuzzy ideals of $R$ for some 2-absorbing fuzzy ideal $\gamma$ of $R$. Then $\mu=\bigcap_{i=1}^{n} \mu_{i}$ is a $\gamma$-2-absorbing primary fuzzy ideal of $R$.

Proof. Suppose that $x_{r} y_{s} z_{t} \in \mu$ and $x_{r} y_{s} \notin \mu$. Then $x_{r} y_{s} \notin \mu_{j}$ for some $n \geq j \geq 1$ and $x_{r} y_{s} z_{t} \in \mu_{j}$ for all $n \geq j \geq 1$. Since $\mu_{j}$ is a $\gamma$-2-absorbing primary fuzzy ideal, we have $y_{s} z_{t} \in$ $\sqrt{\mu_{j}}=\gamma=\bigcap_{i=1}^{n} \sqrt{\mu_{i}}=\sqrt{\bigcap_{i=1}^{n} \mu_{i}}=\sqrt{\mu}$ or $x_{r} z_{t} \in \sqrt{\mu_{j}}=$ $\gamma=\bigcap_{i=1}^{n} \sqrt{\mu_{i}}=\sqrt{\bigcap_{i=1}^{n} \mu_{i}}=\sqrt{\mu}$. Thus, $\mu$ is a $\gamma$-2-absorbing primary ideal of $R$.

In the following example, we show that if $\mu_{1}, \mu_{2}$ are 2absorbing primary fuzzy ideals of a ring $R$, then $\mu_{1} \cap \mu_{2}$ need not to be a 2-absorbing primary fuzzy ideal of $R$.

Example 27. Let $R=Z$, the ring of integers. Define the fuzzy ideals $\mu_{1}$ and $\mu_{2}$ of $Z$ by

$$
\mu_{1}(x)= \begin{cases}1, & x \in 50 Z \\ 0, & x \notin 50 Z\end{cases}
$$

and by

$$
\mu_{2}(x)= \begin{cases}1, & x \in 75 Z \\ 0, & x \notin 75 Z\end{cases}
$$


Here $\mu_{1}$ and $\mu_{2}$ are 2-absorbing primary fuzzy ideals of $Z$ by Proposition 20. But it is not difficult to show that $\mu_{1} \cap \mu_{2}$ is not a 2 -absorbing primary fuzzy ideal of $Z$. Since

$$
\left(\mu_{1} \cap \mu_{2}\right)(x)= \begin{cases}1, & x \in 150 Z, \\ 0, & x \notin 150 Z,\end{cases}
$$

then $25_{1} .3_{1} .2_{1} \in \mu_{1} \cap \mu_{2}$ but $25_{1} 3_{1} \notin \mu_{1} \cap \mu_{2}, 25_{1} 2_{1} \notin \mu_{1} \cap \mu_{2}$, and $2_{1} 3_{1} \notin \mu_{1} \cap \mu_{2}$. Moreover, by the definition of

$$
\sqrt{\mu_{1} \cap \mu_{2}}= \begin{cases}1, & x \in 30 Z, \\ 0, & x \notin 30 Z,\end{cases}
$$

we conclude that $25_{1} \cdot 3_{1} \cdot 2_{1} \in \mu_{1} \cap \mu_{2}$ but $25_{1} 3_{1} \notin \sqrt{\mu_{1} \cap \mu_{2}}$, $25_{1} 2_{1} \notin \sqrt{\mu_{1} \cap \mu_{2}}$, and $2_{1} 3_{1} \notin \sqrt{\mu_{1} \cap \mu_{2}}$. Hence, $\mu_{1} \cap \mu_{2}$ is not a 2-absorbing primary fuzzy ideal of $Z$.

Theorem 28. Let $\mu$ be a fuzzy ideal of $R$. If $\sqrt{\mu}$ is a prime fuzzy ideal of $R$, then $\mu$ is a 2-absorbing primary fuzzy ideal of $R$.

Proof. Assume that $x_{r} y_{s} z_{t} \in \mu$ and $x_{r} y_{s} \notin \mu$ for any $x, y, z \in$ $R$ and $r, s, t \in[0,1]$. Since $x_{r} y_{s} z_{t} \in \mu$ and $R$ is commutative ring, we have $x_{r} y_{s} z_{t} z_{t}=\left(x_{r} z_{t}\right)\left(y_{s} z_{t}\right) \in \mu \subseteq \sqrt{\mu}$. Thus, $x_{r} z_{t} \in$ $\sqrt{\mu}$ or $y_{s} z_{t} \in \sqrt{\mu}$ since $\sqrt{\mu}$ is a prime fuzzy ideal of $R$. Hence, we conclude that $\mu$ is a 2 -absorbing primary fuzzy ideal of $R$.

Corollary 29. If $\mu$ is a prime fuzzy ideal of $R$, then $\mu^{n}$ is 2absorbing primary fuzzy ideal of $R$ for any $n \in Z^{+}$.

Proof. Let $\mu$ be a prime fuzzy ideal and $x_{r} y_{s} z_{t} \in \mu^{n}$ but $x_{r} y_{s} \notin$ $\mu^{n}$ for any $n \in Z^{+}$. Since $x_{r} y_{s} z_{t} \in \mu^{n}$ and $R$ is commutative ring, we conclude that $x_{r} y_{s} z_{t} z_{t}=\left(x_{r} z_{t}\right)\left(y_{s} z_{t}\right) \in \mu^{n} \subseteq \mu$. Hence, $x_{r} z_{t} \in \mu=\sqrt{\mu^{n}}$ or $y_{s} z_{t} \in \mu=\sqrt{\mu^{n}}$ since $\mu$ is prime fuzzy ideal of $R$.

Theorem 30. Let $\left\{\mu_{i}: i \in I\right\}$ be a directed collection of 2absorbing primary fuzzy ideals of $R$. Then the fuzzy ideal $\mu=$ $\bigcup_{i \in I} \mu_{i}$ is a 2-absorbing primary fuzzy ideal of $R$.

Proof. Suppose $x_{r} y_{s} z_{t} \in \mu$ and $x_{r} y_{s} \notin \mu$ for some $x_{r}, y_{s}, z_{t}$ fuzzy points of $R$. Then there are some $j \in I$ such that $x_{r} y_{s} z_{t} \in$ $\mu_{j}$ and $x_{r} y_{s} \notin \mu_{j}$ for all $j \in I$. Since $\mu_{j}$ is 2-absorbing primary fuzzy ideal, we have $y_{s} z_{t} \in \sqrt{\mu_{j}}$ or $x_{r} z_{t} \in \sqrt{\mu_{j}}$. Thus, $y_{s} z_{t} \in$ $\sqrt{\mu_{j}} \subseteq \bigcup_{i \in I} \sqrt{\mu_{i}}=\sqrt{\bigcup_{i \in I} \mu_{i}}=\mu$ or $x_{r} z_{t} \in \sqrt{\mu_{j}} \subseteq \bigcup_{i \in I} \sqrt{\mu_{i}}=$ $\sqrt{\bigcup_{i \in I} \mu_{i}}=\mu$.

Theorem 31. Let $f: R \rightarrow S$ be a ring homomorphism. If $\xi$ is a 2-absorbing primary fuzzy ideal of $S$, then $f^{-1}(\xi)$ is a 2absorbing primary fuzzy ideal of $R$.

Proof. Assume that $x_{r} y_{s} z_{t} \in f^{-1}(\xi)$, where $x_{r}, y_{s}, z_{t}$ are any fuzzy points of $R$. Then $r \wedge s \wedge t \leq f^{-1}(\xi)(x y z)=\xi(f(x y z))=$ $\xi(f(x) f(y) f(z))$. Let $f(x)=a, f(y)=b$, and $f(z)=c \in S$. Thus, we get that $r \wedge s \wedge t \leq \xi(a b c)$ and $a_{r} b_{s} c_{t} \in \xi$. Since $\xi$ is a 2-absorbing primary fuzzy ideal, we conclude that $a_{r} b_{s} \in \xi$ or $a_{r} c_{t} \in \sqrt{\xi}$ or $b_{s} c_{t} \in \sqrt{\xi}$. If $a_{r} b_{s} \in \xi$, then $r \wedge s \leq \xi(a b)=$ $\xi(f(x) f(y))=\xi(f(x y))=f^{-1}(\xi)(x y)$; hence, we conclude that $x_{r} y_{s} \in f^{-1}(\xi)$.

If $a_{r} c_{t} \in \sqrt{\xi}$, then, for some $n \in Z^{+}, r \wedge t \leq \xi\left(a^{n} c^{n}\right)=$ $\xi\left(f(x)^{n} f(z)^{n}\right) \leq \bigvee_{n \geq 1} \xi\left(f(x)^{n} f(z)^{n}\right)=\sqrt{\xi}(f(x) f(z))=$ $\sqrt{\xi}(f(x z))=f^{-1}(\sqrt{\xi})(x z)=\sqrt{f^{-1}(\xi)}$. Thus, we get that $x_{r} z_{t} \in \sqrt{f^{-1}(\xi)}$. By a similar way, it can be see that $y_{s} z_{t} \in$ $\sqrt{f^{-1}(\xi)}$

Theorem 32. Let $f: R \rightarrow S$ be a surjective ring homomorphism. If $\mu$ is a 2-absorbing primary fuzzy ideal of $R$ which is constant on $\operatorname{Ker} f$, then $f(\mu)$ is a 2-absorbing primary fuzzy ideal of $S$.

Proof. Suppose that $a_{r} b_{s} c_{t} \in f(\mu)$, where $a_{r}, b_{s}, c_{t}$ are any fuzzy points of $S$. Since $f$ is a surjective ring homomorphism, there exist $x, y, z \in R$ such that $f(x)=a, f(y)=$ $b, f(z)=c$. Thus, $a_{r} b_{s} c_{t}(a b c)=r \wedge s \wedge t \leq f(\mu)(a b c)=$ $f(\mu)(f(x) f(y) f(z))=f(\mu)(f(x y z))=\mu(x y z)$ because $\mu$ is constant on $\operatorname{Ker} f$. Then we get $x_{r} y_{s} z_{t} \in \mu$. Since $\mu$ is a 2-absorbing primary fuzzy ideal, we conclude $x_{r} y_{s} \in \mu$ or $x_{r} z_{t} \in \sqrt{\mu}$ or $y_{s} z_{t} \in \sqrt{\mu}$.

Thus, $r \wedge s \leq \mu(x y)=f(\mu)(f(x y))=f(\mu)(f(x) f(y))=$ $f(\mu)(a b)$ so $a_{r} b_{s} \in f(\mu)$ or $r \wedge t \leq \sqrt{\mu}(x z)=\bigvee_{n \geq 1} \mu\left(x^{n} z^{n}\right)=$ $\bigvee_{n \geq 1} f(\mu)\left(f\left(x^{n}\right) f\left(z^{n}\right)\right)=\bigvee_{n \geq 1} f(\mu)\left(a^{n} c^{n}\right)=\sqrt{f(\mu)}(a c)$ so $a_{r} c_{t} \in \sqrt{f(\mu)}$.

By a similar way, it is easy to see that $b_{s} c_{t} \in \sqrt{f(\mu)}$ if $y_{s} z_{t} \in$ $\sqrt{\mu}$.

\section{Weakly Completely 2-Absorbing Primary Fuzzy Ideals}

Definition 33. (i) A nonconstant fuzzy ideal $\mu$ of $R$ is called a weakly completely 2-absorbing fuzzy ideal of $R$ if for all $a, b, c \in R, \mu(a b c) \leq \mu(a b)$ or $\mu(a b c) \leq \mu(a c)$ or $\mu(a b c) \leq$ $\mu(b c)$.

(ii) A nonconstant fuzzy ideal $\mu$ of $R$ is called a weakly completely 2-absorbing primary fuzzy ideal of $R$ if for all $a, b, c \in R, \mu(a b c) \leq \mu(a b)$ or $\mu(a b c) \leq \sqrt{\mu}(a c)$ or $\mu(a b c) \leq$ $\sqrt{\mu}(b c)$.

Theorem 34. Every weakly completely 2-absorbing fuzzy ideal of $R$ is a weakly completely 2-absorbing primary fuzzy ideal.

Proof. The proof is straightforward.

The following example shows that the converse of Theorem 34 is not necessarily true.

Example 35. Let $R=Z$, the ring of integers. Define the fuzzy ideal $\mu$ of $Z$ by

$$
\mu(x)= \begin{cases}1, & x \in 8 Z, \\ 0, & x \notin 8 Z .\end{cases}
$$

Assume that $\mu(x y z)>\mu(x y)$ for any $x, y, z \in R$. Thus, $\mu(x y z)=1$ and $0=\mu(x y)$ so we get $x y z \in 8 Z$ and $x y \notin$ 
$8 Z$. Since $8 Z$ is primary ideal of $Z$, we get $z \in 2 Z$. By the definition of radical $\mu$

$$
\begin{aligned}
& \sqrt{\mu}(x)= \begin{cases}1, & x \in 2 Z, \\
0, & x \notin 2 Z,\end{cases} \\
& \sqrt{\mu}(x z)=1, \\
& \sqrt{\mu}(y z)=1,
\end{aligned}
$$

hence, $\sqrt{\mu}(x z) \geq \mu(x y z)$ or $\sqrt{\mu}(y z) \geq \mu(x y z)$. Therefore, $\mu$ is a weakly completely 2 -absorbing primary fuzzy ideal. But since $\mu(2.2 .2)=1>0=\mu(2.2)$, we conclude that $\mu$ is not a weakly completely 2 -absorbing fuzzy ideal.

Proposition 36. Every primary fuzzy ideal of $R$ is a weakly completely 2-absorbing primary fuzzy ideal.

Proof. Let $\mu$ be a primary fuzzy ideal of $R$. Assume that $\mu(x y z)>\mu(x y)$ for any $x, y, z \in R$. Since $\mu$ is primary fuzzy ideal, we conclude $\sqrt{\mu}(z) \geq \mu(x y z)$. Since $\sqrt{\mu}$ is a fuzzy ideal, we have $\sqrt{\mu}(x z) \geq \sqrt{\mu}(z) \geq \mu(x y z)$ or $\sqrt{\mu}(y z) \geq$ $\sqrt{\mu}(z) \geq \mu(x y z)$. So $\mu$ is a weakly completely 2 -absorbing primary fuzzy ideal.

Lemma 37. Let $\mu$ be a fuzzy ideal of $R$. Then $\mu$ is a weakly completely 2-absorbing primary fuzzy ideal of $R$ if and only if $\mu_{t}$ is a 2-absorbing primary ideal of $R$ for all $t \in[0, \mu(0)]$.

Proof. Assume that $x y z \in \mu_{t}$ and $x y \notin \mu_{t}$ for any $x, y, z \in R$. We show that $y z \in \sqrt{\mu_{t}}$ or $x z \in \sqrt{\mu_{t}}$. Note that $\mu(x y z) \geq t>$ $\mu(x y)$. Since $\mu$ is a weakly completely 2 -absorbing primary fuzzy ideal of $R$, we have $\sqrt{\mu}(x z) \geq \mu(x y z) \geq t$ or $\sqrt{\mu}(y z) \geq$ $\mu(x y z) \geq t$. Thus, $x z \in \sqrt{\mu}_{t}=\sqrt{\mu_{t}}$ or $y z \in \sqrt{\mu}_{t}=\sqrt{\mu_{t}}$. So we conclude that $\mu_{t}$ is a 2-absorbing primary ideal of $R$.

Conversely, assume that $\mu_{t}$ is a 2 -absorbing primary ideal of $R$ for all $t \in[0, \mu(0)]$. If $\mu(x y z)>\mu(x y)$ for any $x, y, z \in R$, then there is a $k \in[0, \mu(0)]$ such that $\mu(x y z)=k$ and $k=$ $\mu(x y z)>\mu(x y)$. So $x y z \in \mu_{k}$ and $x y \notin \mu_{k}$. Since $\mu_{k}$ is a 2absorbing primary ideal of $R$, we get that $x z \in \sqrt{\mu_{k}}=\sqrt{\mu}_{k}$ or $y z \in \sqrt{\mu_{k}}=\sqrt{\mu}_{k}$. Hence, $\sqrt{\mu}(x z) \geq k=\mu(x y z)$ or $\sqrt{\mu}(y z) \geq$ $k=\mu(x y z)$. Therefore, $\mu$ a is weakly completely 2 -absorbing primary fuzzy ideal of $R$.

Theorem 38. If $\mu$ is a weakly completely 2-absorbing primary fuzzy ideal of $R$, then $\sqrt{\mu}$ is a weakly completely 2-absorbing fuzzy ideal of $R$.

Proof. If $\mu$ is a weakly completely 2 -absorbing primary fuzzy ideal, then by Lemma $37 \mu_{t}$ is a 2-absorbing primary ideal of $R$ for any $t \in[0, \mu(0)]$. By [5, Theorem 2.2], $\sqrt{\mu_{t}}=\sqrt{\mu}_{t}$ is 2 -absorbing ideal of $R$. Then it is easy to see that from Definition $33 \sqrt{\mu}_{t}$ is a 2-absorbing ideal of $R$ if and only if $\sqrt{\mu}$ is a weakly completely 2 -absorbing fuzzy ideal.

Definition 39. Let $\mu$ be fuzzy ideal of $R$. Then $\mu$ is called a $K$-2-absorbing primary fuzzy ideal of $R$ if for all $x, y, z \in R$, $\mu(x y z)=\mu(0)$ implies that $\mu(x y)=\mu(0)$ or $\sqrt{\mu}(y z)=\mu(0)$ or $\sqrt{\mu}(x z)=\mu(0)$.
Proposition 40. Every weakly completely 2-absorbing primary fuzzy ideal is a K-2-absorbing primary fuzzy ideal.

Proof. Assume that $\mu$ is a weakly completely 2-absorbing primary fuzzy ideal. If $\mu(x y z)=\mu(0)$ for any $x, y, z \in R$, then $\mu(0) \geq \mu(x y) \geq \mu(x y z)=\mu(0)$ or $\mu(0)=\sqrt{\mu}(0) \geq$ $\sqrt{\mu}(x z) \geq \mu(x y z)=\mu(0)$ or $\mu(0)=\sqrt{\mu}(0) \geq \sqrt{\mu}(y z) \geq$ $\mu(x y z)=\mu(0)$ since $\mu$ is a weakly completely 2-absorbing primary fuzzy ideal. Hence, $\mu(x y)=\mu(0)$ or $\sqrt{\mu}(x z)=\mu(0)$ or $\sqrt{\mu}(y z)=\mu(0)$. We conclude that $\mu$ is a $K$-2-absorbing primary fuzzy ideal.

Note that the following example shows that a $K$-2absorbing primary fuzzy ideal need not be a weakly completely 2-absorbing primary fuzzy ideal.

Example 41. Let $R=Z$, the ring of integers. Define the fuzzy ideal $\mu$ of $Z$ by

$$
\mu(x)= \begin{cases}1, & x=0 \\ \frac{1}{2}, & x \in 30 Z-0 \\ \frac{1}{3}, & x \in Z-30 Z .\end{cases}
$$

Then $\mu$ is a $K$-2-absorbing primary fuzzy ideal. But since

$$
\mu(2.3 .5)=\frac{1}{2}>\bigvee\{\mu(2.3), \mu(2.5), \mu(3.5)\}=\frac{1}{3}
$$

or

$$
\begin{aligned}
\mu(2.3 .5) & =\frac{1}{2}>\bigvee\{\sqrt{\mu}(2.3), \sqrt{\mu}(2.5), \sqrt{\mu}(3.5)\} \\
& =\frac{1}{3}
\end{aligned}
$$

then $\mu$ is not a weakly completely 2 -absorbing primary fuzzy ideal.

Corollary 42. Every weakly completely prime fuzzy ideal is a weakly completely 2-absorbing primary fuzzy ideal.

Proof. Since every weakly completely prime fuzzy ideal is primary fuzzy ideal, by Proposition 36 every weakly completely prime fuzzy ideal is a weakly completely 2-absorbing primary fuzzy ideal.

Theorem 43. Every K-2-absorbing fuzzy ideal is a K-2absorbing primary fuzzy ideal.

Proof. The proof is straightforward.

The following example shows that the converse of Theorem 43 is not true.

Example 44. Define the fuzzy ideal $\mu$ of $Z$ by

$$
\mu(x)= \begin{cases}1, & x \in 8 Z, \\ 0, & x \notin 8 Z .\end{cases}
$$


Then $\mu$ is a $K$-2-absorbing primary fuzzy ideal but since $\mu(2.2 .2)=\sqrt{\mu}(2.2)=\bigvee_{n \geq 1} \mu\left(4^{n}\right)=1=\mu(0)$ and $\mu(2.2 .2)=$ $1=\mu(0) \neq \mu(4)=0$, we have that $\mu$ is not a $K$-2-absorbing fuzzy ideal.

Theorem 45. Let $f: R \rightarrow S$ be a ring homomorphism. If $\xi$ is a weakly completely 2-absorbing primary fuzzy ideal of $S$, then $f^{-1}(\xi)$ is a weakly completely 2-absorbing primary fuzzy ideal of $R$.

Proof. Assume that $f^{-1}(\xi)(x y z)>f^{-1}(\xi)(x y)$ for any $x, y$, $z \in R$. Then $f^{-1}(\xi)(x y z)=\xi(f(x y z))=\xi(f(x) f(y) f(z))>$ $f^{-1}(\xi)(x y)=\xi(f(x y))=\xi(f(x) f(y))$. Since $\xi$ is a weakly completely 2 -absorbing primary fuzzy ideal of $S$, we conclude that $\xi(f(x) f(y) f(z))=f^{-1}(\xi)(x y z) \leq$ $\sqrt{\xi}(f(x) f(z))=\sqrt{\xi}(f(x z))=f^{-1}(\sqrt{\xi}(x z))=\sqrt{f^{-1}(\xi)}(x z)$ or $\xi(f(x) f(y) f(z))=f^{-1}(\xi)(x y z) \leq \sqrt{\xi}(f(y) f(z))=$ $\sqrt{\xi}(f(y z))=f^{-1}(\sqrt{\xi}(y z))=\sqrt{f^{-1}(\xi)}(y z)$. Thus, $f^{-1}(\xi)$ is a weakly completely 2 -absorbing primary fuzzy ideal of $R$.

Theorem 46. Let $f: R \rightarrow S$ be a surjective ring homomorphism. If $\mu$ is a weakly completely 2-absorbing primary fuzzy ideal of $R$ which is constant on $\operatorname{Ker} f$, then $f(\mu)$ is a weakly completely 2-absorbing primary fuzzy ideal of $S$.

Proof. Assume that $f(\mu)(a b c)>f(\mu)(a b)$ for any $a, b, c \in$ $S$. Since $f$ is surjective ring homomorphism, $f(x)=a$, $f(y)=b$, and $f(z)=c$ for some $x, y, z \in R$. Thus, $f(\mu)(a b c)=f(\mu)(f(x) f(y) f(z))=f(\mu)(f(x y z))>$ $f(\mu)(a b)=f(\mu)(f(x) f(y))=f(\mu)(f(x y))$. So, as $\mu$ is constant on $\operatorname{Ker} f, f(\mu)(f(x y z))=\mu(x y z)$ and $f(\mu)(f(x y))=$ $\mu(x y)$. This means that $f(\mu)(a b c)=\mu(x y z)>\mu(x y)=$ $f(\mu)(a b)$. Since $\mu$ is a weakly completely 2 -absorbing primary fuzzy ideal of $R$, we have $\mu(x y z)=f(\mu)(f(x) f(y) f(z))=$ $f(\mu)(a b c) \leq \sqrt{\mu}(x z)=f(\sqrt{\mu})(f(x z))=f(\sqrt{\mu})(a c)=$ $\sqrt{f(\mu)}(a c)$ or $\mu(x y z)=f(\mu)(f(x) f(y) f(z))=f(\mu)(a b c) \leq$ $\sqrt{\mu}(y z)=f(\sqrt{\mu})(f(y z))=f(\sqrt{\mu})(b c)=\sqrt{f(\mu)}(b c)$. Hence, $f(\mu)$ is a weakly completely 2 -absorbing primary fuzzy ideal of $R$.

We state the following corollary without proof. Its proof is a result of Theorems 45 and 46.

Corollary 47. Let $f$ be a homomorphism of a ring $R$ onto $a$ ring $S$. Then $f$ induces a one-one inclusion preserving correspondence between the weakly completely 2-absorbing primary fuzzy ideals of $R$ which is constant on Ker $f$ and the weakly completely 2-absorbing primary fuzzy ideals of $S$ in such a way that if $\mu$ is a weakly completely 2-absorbing primary fuzzy ideal of $R$ constant on $\operatorname{Ker} f$, then $f(\mu)$ is the corresponding weakly completely 2-absorbing primary fuzzy ideal of $S$, and if $\xi$ is a weakly completely 2-absorbing primary fuzzy ideal of $S$, then $f^{-1}(\xi)$ is the corresponding weakly completely 2-absorbing primary fuzzy ideal of $R$.

Remark 48. Note that the following diagram shows the transition between definitions of fuzzy ideals:

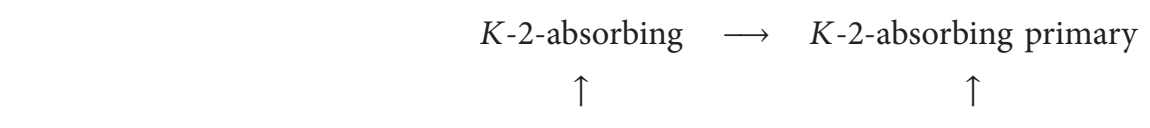

weakly completely prime $\longrightarrow$ w. c. 2-absorbing $\longrightarrow$ w. c. 2-absorbing primary

$\uparrow$

prime $\uparrow$

$\begin{array}{cc}\text { 2-absorbing } & \longrightarrow \\ \text { primary } & \nearrow\end{array}$ $\uparrow$

2-absorbing primary

\section{Conclusion}

This article investigates the weakly completely 2-absorbing primary fuzzy ideal and 2-absorbing primary fuzzy ideal as a generalization of primary fuzzy ideal in commutative rings. Also some characterizations of 2 -absorbing primary fuzzy ideal are obtained. Moreover, we see that a 2-absorbing primary fuzzy ideal by a 2-absorbing primary ideal of a commutative ring is established, so the transition between the two structures can be analyzed.

\section{Conflicts of Interest}

The authors declare that they have no conflicts of interest.

\section{References}

[1] L. A. Zadeh, "Fuzzy sets," Information and Control, vol. 8, no. 3, pp. 338-353, 1965.
[2] W. J. Liu, "Fuzzy invariant subgroups and fuzzy ideals," Fuzzy Sets and Systems, vol. 8, no. 2, pp. 133-139, 1982.

[3] T. K. Mukherjee and M. K. Sen, "Prime fuzzy ideals in rings," Fuzzy Sets and Systems, vol. 32, no. 3, pp. 337-341, 1989.

[4] A. Badawi, "On 2-absorbing ideals of commutative rings," Bulletin of the Australian Mathematical Society, vol. 75, no. 3, pp. 417-429, 2007.

[5] A. Badawi, U. Tekir, and E. Yetkin, "On 2-absorbing primary ideals in commutative rings," Bulletin of the Korean Mathematical Society, vol. 51, no. 4, pp. 1163-1173, 2014.

[6] T. K. Mukherjee and M. K. Sen, "Primary fuzzy ideals and radical of fuzzy ideals," Fuzzy Sets and Systems, vol. 56, no. 1, pp. 97-101, 1993.

[7] V. N. Dixit, R. Kumar, and N. Ajmal, "Fuzzy ideals and fuzzy prime ideals of a ring," Fuzzy Sets and Systems, vol. 44, no. 1, pp. 127-138, 1991. 
[8] F. I. Sidky and S. A. Khatab, "Nil radical of fuzzy ideal," Fuzzy Sets and Systems, vol. 47, no. 1, pp. 117-120, 1992.

[9] F. Callialp, E. Yetkin, and U. Tekir, "On 2-absorbing primary and weakly 2 -absorbing primary elements in multiplicative lattices," Italian Journal of Pure and Applied Mathematics, no. 34, pp. 263276, 2015. 


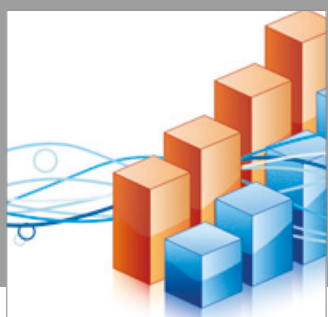

Advances in

Operations Research

vatersals

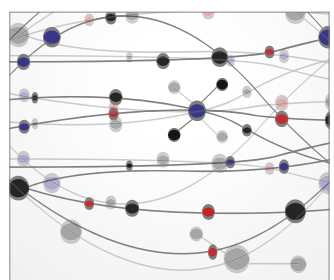

\section{The Scientific} World Journal
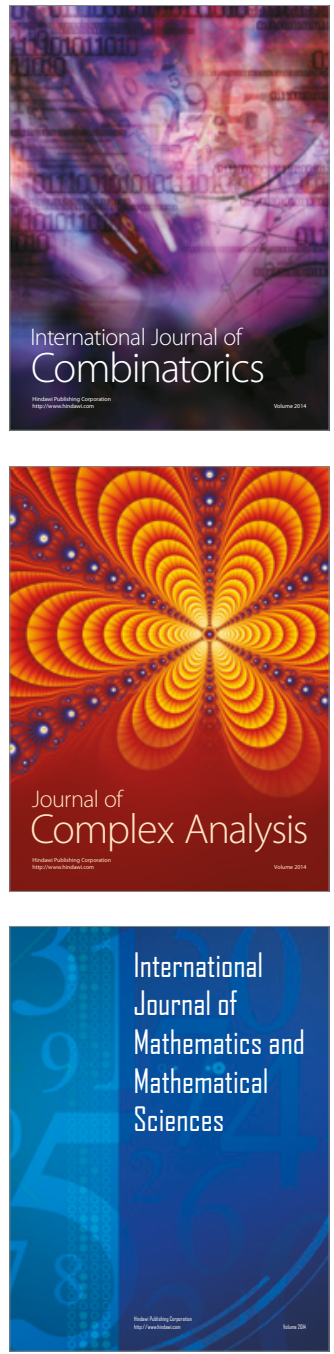
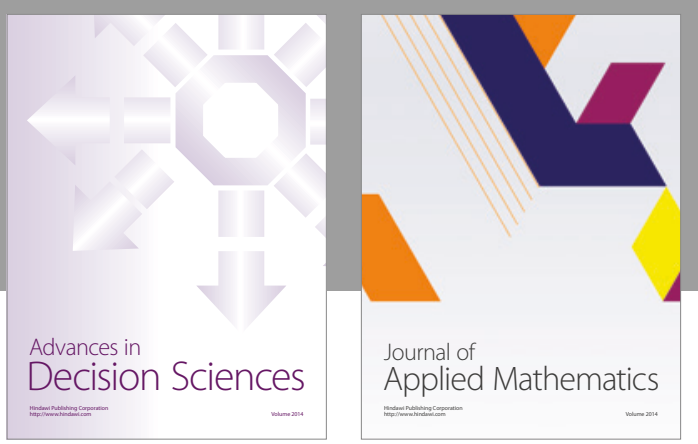

Algebra

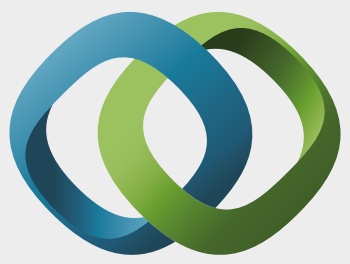

\section{Hindawi}

Submit your manuscripts at

https://www.hindawi.com
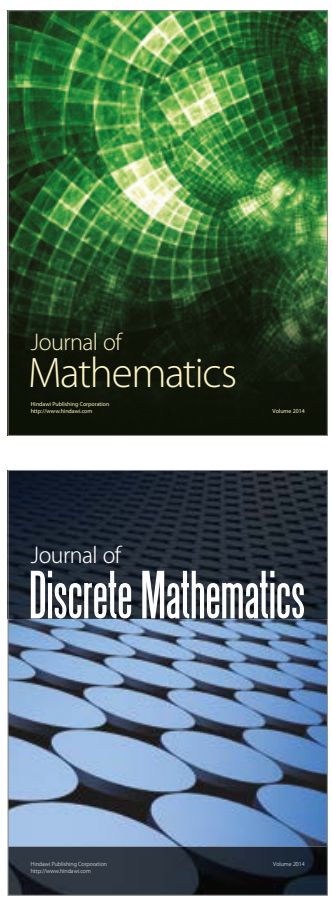

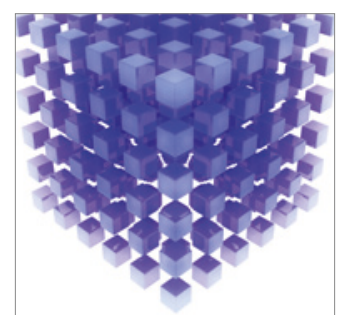

Mathematical Problems in Engineering
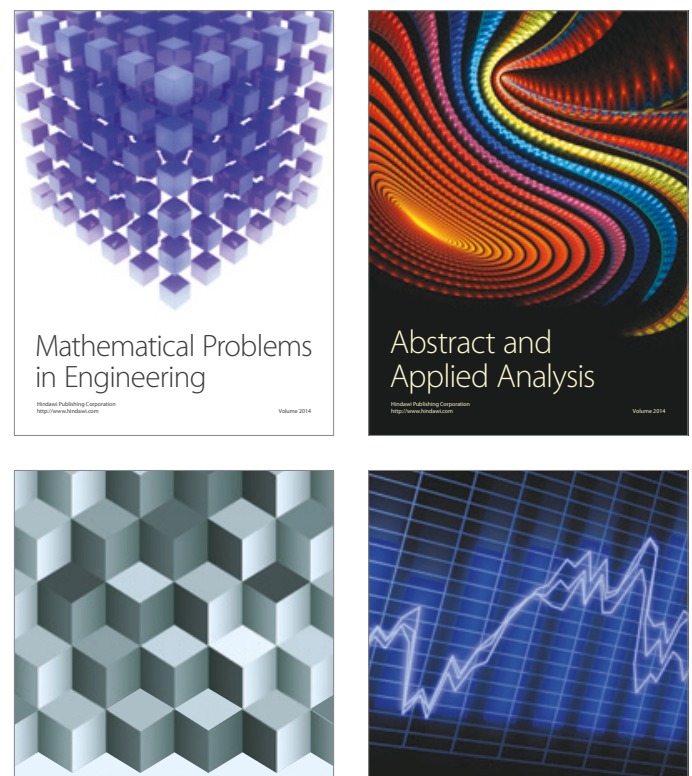

Journal of

Function Spaces

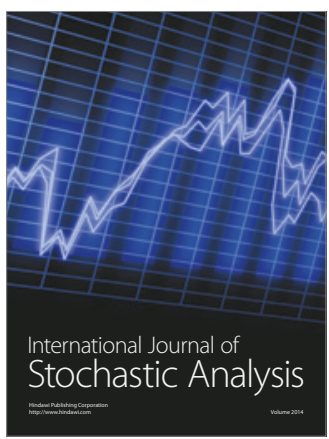

Probability and Statistics
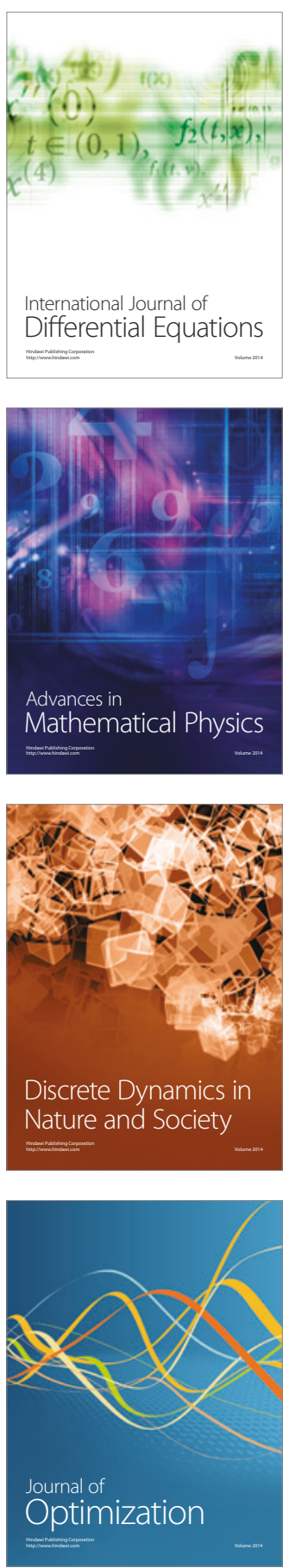